\title{
Haptic Landmark Positioning and Automatic Landmark Transfer in 4D Lung CT Data
}

\author{
Matthias Färber ${ }^{1}$, Björn Gawenda ${ }^{1,2}$, Christian-Arved Bohn² ${ }^{2}$ Heinz Handels ${ }^{1}$ \\ ${ }^{1}$ Department of Medical Informatics, University Medical Center Hamburg-Eppendorf \\ ${ }^{2}$ Computergraphics \& Virtual Reality, University of Applied Sciences Wedel \\ m.faerber@uke.uni-hamburg.de
}

\begin{abstract}
Manual landmark positioning in volumetric image data is a complex task and often results in erroneous landmark positions. The landmark positioning tool presented uses image curvature features to precompute suitable candidates for landmark positions on surface data of anatomical structures. A force-feedback I/O device is then used to haptically guide the user during the definition of the correct landmarks in the 3D data volume. Furthermore, existing landmarks in a time-point of a sequence of $3 \mathrm{D}$ volumes ( $4 \mathrm{D}$ data set) can iteratively be transferred to other time-points using a surface based registration technique. First results show significant time savings and small interobserver variability (IROV) compared to the IROV of manually defined landmark positions using orthogonal slices of the image data.
\end{abstract}

\section{Introduction}

Medical landmarks are definite points in human anatomy that can be described using medical terminology (e.g. bifurcatio tracheae) or unique location definitions (e.g. "apex of the left lung"). They are required to determine quantitative measures like distances or angles, they represent significant points for navigation purposes and can be used for further image processing (e.g. landmark based registration). In this work landmarks are determined in 4D CT lung data to quantify motion of lung structures due to breathing motion. Usually the landmark positions are defined manually using orthogonal (sagittal, coronal, axial) slices of the image data. As orientation and navigation in 3D image data volumes is a complex task even for experienced medical experts, the correct manual positioning of landmarks is a time-consuming process and it often results in misplaced landmarks. For these reasons a tool has been developed to support and guide the user during the positioning and to transfer landmarks between 3D volumes of a $4 \mathrm{D}$ data set.

Previous work in the direction of automatic detection of landmarks has been presented in $[1,2]$. The detection of landmark candidates in our work is based on the work of Hartkens et al. [3], and some ideas of Ehrhardt et al. [4] are used for landmark transfer between time-points. A technique for haptic guidance during the interactive positioning of landmarks has not yet been proposed. 


\section{Materials and Methods}

The assistance during the positioning of landmarks on surface models is split up into three parts: The automatic detection of landmark candidates (2.1), the haptic guidance to these candidates (2.2) and the transfer of landmarks between time-points of a $4 \mathrm{D}$ data set (2.3).

\subsection{Detection of Landmark Candidates}

This work is focussed on the detection of landmarks that lie at bifurcations of the bronchus tree. We restrict the landmark positions to the surface of this organ. Therefore these landmarks are located at saddle points of hyperbolic shaped surfaces and can further be characterized by a strong image curvature. In a first step different measures for surface curvature (Gaussian curvature, mean curvature, and moment based curvature [4]) have been evaluated to detect the saddle points. It turned out, that Gaussian and mean curvature strongly depend on the quality and smoothness of the surface meshes and therefore are inappropriate to detect the landmarks. The moment based curvature approach is more stable to quality issues but has some disadvantages in discriminating saddle points and points on flat surfaces. A combination of moment based and Gaussian curvature where candidates have been defined at positions with minimal Gaussian and small moment based curvature showed better results but was still depending on surface smoothing.

To be independent of surface quality and smoothness it was decided to use an image curvature measure instead. According to the results presented in Hartkens et al. [3] the Rohr operator $k_{\text {Rohr }}$ is used to calculate the image curvature. It is defined by the determinant $\operatorname{det} \underline{C}$ of the structure tensor $\underline{C}$ which is built of the mean image gradients $\nabla g=\left(g_{x}, g_{y}, g_{z}\right)^{T}$ of a local neighborhood in a three-dimensional image $g(x, y, z)$ :

$$
\underline{C}=\overline{\nabla g(\nabla g)^{T}}
$$

Fig. 1(a) shows the results of the Rohr operator transferred to the bronchus surface. The maxima of this measure are located at the bifurcations of the bronchus tree. The resulting landmark candidates (Fig. 1(b)) are computed in a local distance around the surface and therefore incorporate points with a high $k_{\text {Rohr }}$ that do not exactly lie on the surface. Each candidate features a $k_{\text {Rohr }}$ of at least $30 \%$ of the maximum $k_{\text {Rohr }}$ of the entire bronchus area. Furthermore, only the points with highest $k_{\text {Rohr }}$ in a local neighborhood $(3 \times 3 \times 3)$ are chosen.

\subsection{Force Calculation for Haptic Guidance}

A haptic I/O-device (Sensable Phantom Omni) is used to place the landmarks on the organ surface. The visualization toolkit (VTK) and Sensable's OpenHaptics library have been combined, to enable haptic feedback from surface objects that 
are graphically rendered through the VTK-pipeline [5]. Thus the user is able to touch the surface with the haptic device to place landmarks on it.

Furthermore, magnetic forces $\boldsymbol{f}_{m i}$ between the landmark candidates $\boldsymbol{p}_{l i}$ and the device tip $\boldsymbol{p}_{\boldsymbol{t}}$ are rendered around the landmark candidates within local areas with radius $r$ and center points $\boldsymbol{p}_{l i}$ :

$$
\boldsymbol{f}_{m i}=\left\{\begin{array}{l}
k \cdot \boldsymbol{v}_{n} \cdot \frac{|\boldsymbol{v}|^{2}}{r^{2}}, \text { if }|\boldsymbol{v}|<r \\
k \cdot \boldsymbol{v}_{n} \cdot \frac{r^{2}}{|\boldsymbol{v}|^{2}}, \text { if }|\boldsymbol{v}|>=r
\end{array} \text { where } \boldsymbol{v}=\boldsymbol{p}_{t}-\boldsymbol{p}_{l i} \text { and } \boldsymbol{v}_{n}=\frac{\boldsymbol{v}}{|\boldsymbol{v}|}\right.
$$

These forces guide the user to the exact positions of landmark candidates when the device enters the area around a candidate $\boldsymbol{p}_{l i}$. The first case in eq. $2(|\boldsymbol{v}|<r)$ is used to avoid vibration artifacts when the device reaches a landmark candidate. The magnetic forces $\boldsymbol{f}_{m i}$ of all candidates are summed up to build a resulting force $\boldsymbol{F}=\sum_{i} \boldsymbol{f}_{m i} \boldsymbol{F}$ is then added to the surface rendering force.

\subsection{Automatic Landmark Transfer}

Landmarks in a 4D data set are traced by iteratively transferring the existing landmarks in the image volume of one time-point to the following image volumes. A non-linear surface based registration approach [4] is used to transfer the landmarks by matching the area around a given landmark (surface patch) to a corresponding area on the destination surface of the next time-point. Supposed a landmark $\boldsymbol{p}_{l}$ is placed on a triangulated surface patch consisting of $n$ vertices $\boldsymbol{p}_{s, i}$. This surface patch is then deformed to match a set of vertices $\boldsymbol{p}_{d, i}$ that build up the corresponding surface patch at the next time-point. Surface constrained diffusion [6] which is based on Gaussian smoothed displacement fields is used to iteratively deform the surface patch. Corresponding vertices are found using a distance measure $d$ that is a weighted sum of the Euclidean distance $d_{e}=\left|\boldsymbol{p}_{d}-\boldsymbol{p}_{d}\right|$, the distance of the normals $d_{n}=-\frac{\boldsymbol{n}_{s} \cdot \boldsymbol{n}_{d}}{2}+0.5$, and the distances

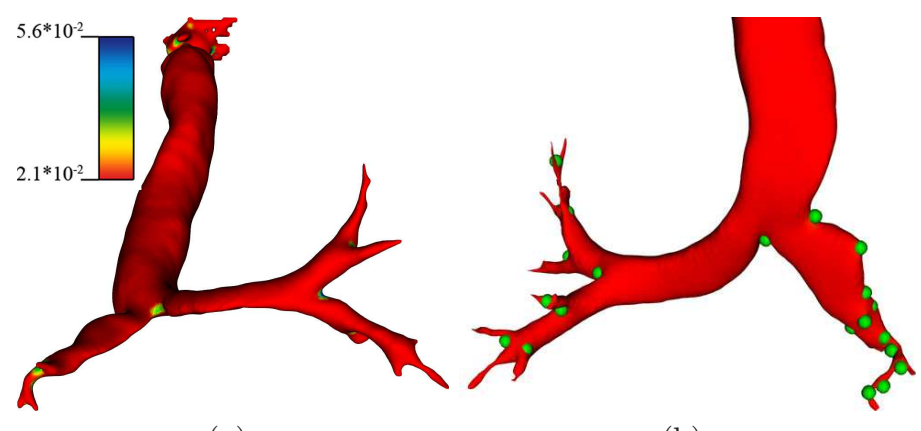

(a)

(b)

Fig. 1. (a) Rohr operator results on the bronchus surface. (b) Back of the bronchus showing the resulting landmark candidates 
Table 1. Standard deviations $(\sigma)$ and mean distance $\bar{d}$ between landmarks of different users (IROV). $\bar{d}_{h m}$ : Mean distance between manually and "haptically" placed landmarks (IAOV)

\begin{tabular}{|c|c|c|c|c|c|c|c|c|c|}
\hline \multirow[b]{2}{*}{ ID } & \multicolumn{4}{|c|}{ haptically (in $\mathrm{mm}$ ) } & \multicolumn{4}{|c|}{ manually (in $\mathrm{mm}$ ) } & \multirow[b]{2}{*}{$\bar{d}_{h m}$} \\
\hline & $\sigma_{x}$ & $\sigma_{y}$ & $\sigma_{z}$ & $\bar{d}$ & $\sigma_{x}$ & $\sigma_{y}$ & $\sigma_{z}$ & $\bar{d}$ & \\
\hline 0 & 0.561 & 1.159 & 0.623 & 1.106 & 0.977 & 0.488 & 3.326 & 2.687 & 0.47 \\
\hline 1 & 0.957 & 1.106 & 1.384 & 1.627 & 3.937 & 3.686 & 13.500 & 11.188 & 4.39 \\
\hline 2 & 0.206 & 0.803 & 0.521 & 0.686 & 1.229 & 0.977 & 2.839 & 2.401 & 1.53 \\
\hline 3 & 0.675 & 0.688 & 0.526 & 0.854 & 4.980 & 13.351 & 5.612 & 13.026 & 10.39 \\
\hline 4 & 1.099 & 1.005 & 0.567 & 1.158 & 6.398 & 4.809 & 6.364 & 8.728 & 8.85 \\
\hline 5 & 1.266 & 0.680 & 1.119 & 1.485 & 6.404 & 13.493 & 10.247 & 15.633 & 12.14 \\
\hline
\end{tabular}

of the image curvature $k_{\text {Rohr }}$ at the vertex positions $d_{c}=\left|k_{\operatorname{Rohr}}\left(\boldsymbol{p}_{d}\right)-k_{\mathrm{Rohr}}\left(\boldsymbol{p}_{s}\right)\right|$ :

$$
d=\omega_{e} \cdot d_{e}+\omega_{n} \cdot d_{n}+\omega_{c} \cdot d_{c}
$$

The resulting displacement of the landmark $\boldsymbol{p}_{\boldsymbol{l}}$ is then derived by interpolating the displacement vectors of the corners of the underlying triangle of $\boldsymbol{p}_{\boldsymbol{l}}$.

\subsection{Evaluation}

A first evaluation has been done by placing landmarks of the bronchus tree in three time-points of a 4D lung CT data set. Four users with different medical experience manually positioned 6 landmarks using orthogonal slice-views of the volumetric image data at three time-points. Then each user placed the landmarks of the first time-point on the surface of the bronchus guided by the haptic device. These landmarks have been iteratively transferred to the next time-points allowing the user to correct misplaced landmarks using the haptic device.

\section{Results}

The time used to manually position all required landmarks took 30 to $35 \mathrm{~min}$. compared to 7 to $10 \mathrm{~min}$. consumed for haptically guided positioning and landmark transfer. The time to segment the bronchus and to generate the required surface models took about $10 \mathrm{~min}$. Tab. 1 shows the interobserver variability (IROV) for haptically and manually placed landmarks $\left(\sigma_{y}\right.$ and $\left.\bar{d}\right)$ and the mean intraobserver variability (IAOV) as mean distance between the corresponding landmarks of each user $\bar{d}_{h m}$. The results of Tabelle 1 are supported by user opinions that conclude a higher intuitiveness during the guided positioning.

\section{Discussion}

The results show that it is hard to find the correct positions for some landmarks (especially no. 1, 3, and 5, which are located deep in the bronchus tree), when no 
Fig. 2. GUI of the landmark positioning tool showing orthogonal slices of the image data (right) and a $3 \mathrm{D}$ visualization overlaid with an image of the haptic device

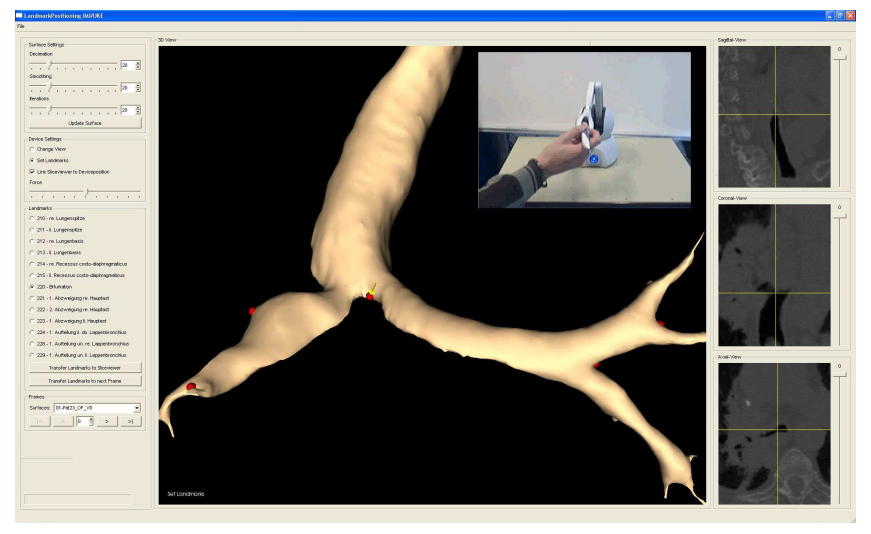

haptic guidance can be used. Therefore the landmark positioning tool presented forms an intuitive and reproducible way of placing landmarks of lung structures in 4D data. It offers significant time-savings and smaller inter- and intraobserver variability than the manual alternative. The guided navigation with the haptic device makes it easy to find the correct landmark positions and the transfer to the next time-point reduces further interaction time.

A restriction of our method is the need for surface models of the lung structures which require a segmentation. In case of the bronchus this can be done nearly automatically using volume growing. But as segmentation is not always such an easy task, our plans for the future include to make the tool independent from surface models using volume rendering techniques.

\section{References}

1. Wörz S, Rohr K. Localization of anatomical point landmarks in 3D medical images by fitting 3D parametric intensity models. Med Image Anal. 2006;10(1):41-58.

2. Berlinger K, Roth M, Sauer O, et al. Fully automatic detection of corresponding anatomical landmarks in volume scans of different respiratory state. Med Phys. 2006;33(6):1569-72.

3. Hartkens T, Rohr K, Stiehl HS. Evaluation of 3D operators for the detection of anatomical point landmarks in MR and CT images. Comput Vis Image Underst. 2002;86(2):118-36.

4. Ehrhardt J, Handels H, Plötz W, et al. Atlas-based recognition of anatomical structures and landmarks and the automatic computation of orthopedic parameters. Methods Inf Med. 2004;43(4):391-7.

5. Färber M, Drescher F, Ehrhardt J, et al. Integration von haptischen Ein-/Ausgabegeräten zur intuitiven Interaktion mit virtuellen Körpern in OP-Planungssysteme. Proc GMDS. 2006; p. 71-2.

6. Andresen PR, Nielsen M. Non-rigid registration by geometry-constrained diffusion. Med Image Anal. 2001;5(2):81-8. 